\title{
BIASES IN MASS ESTIMATES OF GROUPS OF GALAXIES
}

\author{
Gary A. Mamon \\ New York University
}

The knowledge of the masses of small groups of galaxies is important, because the timescales for collapse and virialization of groups depend on their mass-density. While the large inefficiency of the virial mass statistic is well known (Bahcall and Tremaine 1981, hereafter BT, and references therein), biases in the virial mass may produce wrong evolution timescales for small groups. These biases originate from group contamination by interlopers, and from the different galaxy and dark matter distributions inside the groups (caused by mass segregation). This second bias was first studied by Smith $(1980,1984)$ although already implicit in the work of Limber (1959). It formally arises because the ratio $2 T / C$ where $C=\sum \mathbf{F}_{\alpha} \cdot \mathbf{R}_{\alpha}$ (the Clausius virial) is not the same for the luminous and global matter distributions. We illustrate here some quantitative aspects of this Limber bias from the output of $N$-body simulations of groups of 8 galaxies described elsewhere (Mamon 1986).

Table 1 below shows for the projected groups the median ratios of virial (luminosity weighted) and projected (BT) mass to total mass interior to the smallest sphere containing the centers of the galaxies.

Table 1

\begin{tabular}{lcccccc}
\hline \hline $\begin{array}{c}\text { Initial } \\
\text { Conditions }\end{array}$ & $\begin{array}{c}\text { Group } \\
\text { Density }\end{array}$ & $\begin{array}{c}\text { Galaxy } \\
\text { Type }\end{array}$ & $\begin{array}{c}\text { Time } \\
(\mathrm{Gyr})\end{array}$ & $\begin{array}{c}\text { \# in } \\
\text { sample }\end{array}$ & $M_{\text {vir }} / M$ & $M_{\text {pro }} / M$ \\
\hline Virialized & Dense & Halo & 0.5 & 216 & 0.99 & 1.12 \\
Virialized & Dense & Hubble & 2 & 111 & 0.94 & 1.10 \\
Virialized & Loose & Halo & 20 & 267 & 0.89 & 1.01 \\
Virialized & Loose & Hubble & 20 & 264 & 0.81 & 0.91 \\
Collapsing & Loose & Halo & 20 & 195 & 0.98 & 0.87 \\
Collapsing & Loose & Hubble & 20 & 231 & 0.95 & 1.00 \\
\hline
\end{tabular}

The numbers in Table 1 indicate that the median virial mass is a fair mass estimator, although it is generally too low by $10 \%$. The projected mass also produces consistent values with a constant of $32 /(\pi G N)$ in front of the sum of BT's equation (20). A similar result is reached by Heisler et al. (1986). The softening of the potential energy into a Clausius virial for dense groups of often overlapping galaxies has a negligible effect on the median mass estimate. Restricting the analysis to groups or subgroups of 4 galaxies produces similar results.

Bahcall, J. N. and Tremaine, S. 1981, Ap. J., 244, 805.

Heisler, J., Tremaine, S., and Bahcall, J. N. 1986, preprint, and in this volume.

Limber, 1959, Ap. J., 130, 414.

Mamon, G. A. 1986, to be submitted to Ap. J..

Smith, H. 1980, Ap. J., 241, 63; 1984, in Custers and Groups of Galaxies, p. 523. 